\title{
Manipulation and the Causal Markov Condition
}

\author{
Daniel Hausman and James Woodward $†$
}

This paper explores the relationship between a manipulability conception of causation and the causal Markov condition (CM). We argue that violations of $\mathrm{CM}$ also violate widely shared expectations - implicit in the manipulability conception-having to do with the absence of spontaneous correlations. They also violate expectations concerning the connection between independence or dependence relationships in the presence and absence of interventions.

1. Introduction. The causal Markov condition (CM) relates probability distributions to the causal structures that generate them. Given the direct causal relationships among the variables in some set $\mathbf{V}$ and an associated probability distribution $P$ over $\mathbf{V}, \mathrm{CM}$ says that conditional on its parents (its direct causes in $\mathbf{V}$ ) every variable is independent of every other variable, except its effects. Writing $p a_{i}$ for the parents of $X_{i}$ and " $X_{i} \perp$ $X_{j} / p a_{i}$ " for " $X_{i}$ and $X_{j}$ are probabilistically independent conditional on $p a_{i} "$

CM. For all $X_{i}, X_{j}, i \neq j$ in $\mathbf{V}$, if $X_{i}$ does not cause $X_{j}$, then $X_{i} \perp$ $X_{j} / p a_{i}$.

$\mathrm{CM}$ is a generalization of familiar screening off conditions. CM implies that the full set of common causes screens off joint effects from one another, that direct causes screen off effects from distal causes, and that variables that are not related as cause and effect or as effects of a common cause are probabilistically independent.

$\mathbf{C M}$ may fail if the variable set $\mathbf{V}$ is not acyclic or omits common causes, if variables are not distinct in the right way, if variables are too coarse grained, if the probability distribution $P$ is a mixture of distinct distri-

$\dagger$ To contact the authors, please write to: Daniel Hausman, University of Wisconsin, Department of Philosophy, 600 N. Park Street, Madison, WI 53706; e-mail: dhausman@wisc.edu. James Woodward, California Institute of Technology, Humanities \& Social Sciences 101-40, Pasadena, CA 91125-0001; e-mail: jfw@hss.caltech.edu.

Philosophy of Science, 71 (December 2004) pp. 846-856. 0031-8248/2004/7105-0016\$10.00 Copyright 2004 by the Philosophy of Science Association. All rights reserved. 
butions resulting from different causal structures, or if the population in which $P$ holds is selected by conditioning on a common effect of variables in $\mathbf{V}$, or is biased or unrepresentative in some other way. In what follows, we assume that none of these "defeating" conditions obtain.

Given the absence of these defeating conditions, what reasons are there to suppose that CM holds? When causal relationships are pseudo-indeterministic and the "error terms" are probabilistically independent of one another and of the other causal variables in the equations in which they appear, CM must hold. (Pseudo-indeterminism holds when the underlying causal relationships are deterministic, but for each variable $X_{i}$ in $\mathbf{V}$, some direct causes of $X_{i}$, the combined effect of which is represented by an additive error term $U_{i}^{\prime}$, are omitted from $\mathbf{V}$.) However, this observation says nothing about whether $\mathrm{CM}$ holds in indeterministic contexts, and at least one prominent critic, Nancy Cartwright, maintains that CM is frequently violated under indeterminism. Moreover, the rationale for the independence assumptions described above is that variables are probabilistically independent if they are not related as cause and effect or as effects of a common cause, which is itself an implication of CM. Those who question $\mathrm{CM}$ are likely to question these assumptions. Finally, one would like some insight into whether any widely recognized features of causation make CM especially natural.

In this essay we argue for an intimate connection between a manipulability conception of causation and CM. We focus on this connection because, first, manipulation is crucial to our conception of causation and to the contrast between causation and mere correlation. When $X$ and $Y$ are correlated and $X$ does not cause $Y$, one expects that when one manipulates $X$, the correlation will break down. By contrast, if $X$ causes $Y$, one expects that for some range of values of $X$, if one is able to manipulate those values, one can thereby control the value of $Y$. Second, both graphical and equational representations of causal relationships assumed as background to $\mathrm{CM}$ are often given a interventionist interpretation. For example, theorists take the presence of an arrow directed from $X$ to $Y$ (indicating that $X$ is a direct cause of $Y$ ) to mean that if $X$ were to be appropriately manipulated while all other variables in the graph were held fixed, the value of $Y$ would change (Glymour 1997 and Woodward 2003). Are there any reasons to expect CM to hold when "cause" is given such a manipulability interpretation?

2. Interventions, Modularity, and Causation. Some philosophers have attempted to use the connection between causation and manipulation to provide a reductive analysis of causation. We think this is a hopeless undertaking, because causal notions are required to explain what an in- 
tervention is. Nonetheless, the connection between causation and manipulation can be genuinely illuminating.

The connection is not simple. It is not true that $X$ causes $Y$ if and only if every intervention on $X$ results in some change in $Y$. Consider threshold effects: even though $X$ causes $Y$, an intervention that changes the value of $X$ from $x$ to $x^{*}$ may be "too small" to bring about a change in the value of $Y$. In addition, $X$ may have causal effects on $Y$ along different paths, which exactly cancel. In such failures of "faithfulness" (Spirtes, Glymour, and Scheines 2000), no intervention on $X$ will change the value of $Y$, even though $X$ is, in the terminology of Hitchcock 2001 and Woodward 2003, a contributing cause of $Y$. Moreover, some interventions on $X$ may be outside the "domain of invariance" of the relationship between $X$ and $Y$ (see below). Finally, if $X$ is an indeterministic cause of $Y$, then interventions on $X$ may fail to change the value of $Y$, although, unless one faces one of the other difficulties just described, interventions on $X$ should change $Y$ 's probability distribution.

There is nevertheless a plausible sufficient condition which for reasons that emerge shortly, we call MOD*: If some intervention with respect to $X_{i}$ changes the probability distribution of some other variable $X_{j}$, then $X_{i}$ causes $X_{j}$. MOD* is obviously not an analysis of causation. MOD* states only a sufficient condition, and it relies on the notion of an intervention which will be given an explicitly causal characterization.

Before turning to this characterization, we need to make our framework more explicit and take account of some complications. When causation is deterministic, a system of causal relationships may be represented by a set of equations of the form: $X_{i}=f_{i}\left(p a_{i}, U_{i}^{\prime}\right)$ for each variable $X_{i}$ in the variable set $\mathbf{V} ; p a_{i}$ are the parents of $X_{i}$ and $U_{i}^{\prime}$ represents the action of causes of $X_{i}$ omitted from $\mathbf{V}$. Within this framework, if the value of $X_{i}$ is "set" by an intervention and the equation system correctly predicts whether (and how) the values of all other variables change, then the equation system is "causally correct": it correctly describes the quantitative causal relationship between $X_{i}$ and all the other variables. When an equation system is causally correct, one may derive the outcome of an intervention with respect to $X_{i}$ by replacing the equation in which $X_{i}$ is a dependent variable with a new equation specifying the value to which $X_{i}$ was set and leaving all the other equations alone. Obviously, this procedure will work reliably only if interventions that disrupt the relations between $X_{i}$ and its parents do not alter other causal relationships in the system and the equations that govern them. In other words, equations not containing the variable $X_{i}$ as a dependent variable should be invariant-left unchanged - under interventions that set the value of $X_{i}$.

In earlier work (1999), we said that a set of equations meeting this invariance condition was "modular." The idea is if a set of equations is 
causally correct, separate equations should represent distinct causal mechanisms or "modules," and if these are distinct it should be possible to interfere with each separately without disrupting the others. Modularity fits with a manipulability conception of causation: If an equation in which $X_{j}$ is the dependent variable changes whenever there is an intervention with respect to $X_{i}$, then according to a manipulability view of causation there is a causal relationship between $X_{i}$ and $X_{j}$, and the equation system fails to specify it correctly.

However, there is a complication: as noted above, causal relations are not invariant to every intervention. Consider a simple spring whose restoring force $F$ for displacement $Y$ is

$$
F=-k Y \quad\left(\text { for } 0 \leq Y \leq y^{*}\right) .
$$

(1) describes the spring's behavior if it is not stretched too far. If $Y>$ $y^{*}$, the restoring force no longer increases proportionally to the displacement. (1) is invariant to only some interventions with respect to $Y$. Suppose that one replaces (1) with

$$
\begin{aligned}
& F=-k Y \text { for } 0 \leq Y \leq y^{*} ; \\
& F=-g(Y) \text { for } y^{*}<Y \leq y^{* *} ; \\
& F=0 \text { for } Y>y^{* *} .
\end{aligned}
$$

$y^{* *}$ is the point when the spring breaks. Even if $\left(1^{\prime}\right)$ correctly predicts the restoring force for springs that have not previously been stretched beyond $y^{*}$, stretching the spring beyond $y^{*}$ will distort it in such a way that $\left(1^{\prime}\right)$ will no longer correctly describe the restoring force for extensions less than $y^{*}$. Moreover, once $Y>y^{* *}, F=0$ for all subsequent extensions.

One might try to accommodate this fact by writing down a yet more complicated set of equations ( $\left.1^{\prime \prime}\right)$ incorporating information about the previous history of the spring. However, even with such temporal qualifying clauses, $\left(1^{\prime \prime}\right)$ will still not provide correct predictions regarding all interventions - for example, $\left(1^{\prime \prime}\right)$ will break down under manipulations of the extension under extreme heat. In systems such as the spring, we know of no causal equations that are invariant under all interventions.

Accordingly, we cannot accept Nancy Cartwright's proposal (in her criticism of Hausman and Woodward 1999) that the modularity requirement be built into the characterization of an intervention (Cartwright, 2002). She suggests that a manipulation of $X_{i}$ should not count as a bonafide intervention on $X_{i}$ unless it leaves undisturbed all causal relationships in the system of interest, except of course the relationship between $X_{i}$ and its parents. Cartwright is worried by the possibility that a putative intervention on $X_{i}$ (like an extension that breaks the spring) may disrupt the connection between $X_{i}$ and its effects. If there is no change in an effect 
$X_{j}$ of $X_{i}$ under such a disrupting intervention, one might mistakenly conclude that $X_{i}$ does not cause $X_{j}$.

But the proper response is not to build modularity into the definition of an intervention. Doing so trivializes the connection between invariance under interventions and causal correctness (since the notion of an intervention builds in reference to the correct set of causal relationships) and consequently prevents one from using the notion of invariance under interventions (or the behavior of $X_{j}$ under interventions on $X_{i}$ ) to provide any independent purchase on what it is for an equation linking $X_{i}$ to $X_{j}$ to be causally correct. Moreover, as examples like (1)-(1") above illustrate, what most people would regard as interventions with respect to actual causal systems are invariant only to some range of interventions. Finally, the proposal requires that the notion of an intervention be relativized to a specific set of functional relationships: the same manipulation of $Y$ may be an intervention with respect to (1) but not with respect to $\left(1^{\prime}\right)$. The proper response to Cartwright's worry that a manipulation that destroys the connection between $X_{i}$ and its effects will lead to mistaken causal conclusions is to recognize that causal relationships have "domains of invariance" - ranges of interventions over which they continue to hold, even though they do not hold universally. Modularity should be understood as claiming that for each $X_{i}$ there will be some range of interventions (interventions that are of the "right kind" and not "too big") that disrupt only the relationship between $X_{i}$ and its direct causes and no others in the system of interest. We take this to be a substantive claim concerning the nature of causation, which is grounded in a manipulability view.

So far we have been talking only about deterministic causal relationships. When causation is indeterministic, we shall assume that for each $X_{i}$ in $\mathbf{V}, \mathrm{P}\left(X_{i}\right)=g_{i}\left(p a_{i}, U_{i}^{\prime}\right)$, again where $g_{i}$ is some function and $U_{i}^{\prime}$ a set of omitted causes that bears no causal relations to any other variables in $\mathbf{V}$ apart from those that result from its causal relation to $X_{i}$. That is, when causation is indeterministic, the probability distribution of $X_{i}$ is determined by the values of its parents and $U_{i}^{\prime}$. We will return to the status of this "determinism of probabilities" assumption below. Within this indeterministic framework there is a natural analogue to the modularity condition: a system of equations of the form $\mathrm{P}\left(X_{i}\right)=g_{i}\left(p a_{i}, U_{i}^{\prime}\right)$ will be modular if for each variable $X_{i}$, there exists a range of interventions that disrupt only the equation linking $X_{i}$ to its parents and $U_{i}^{\prime}$-i.e., only the function $g_{i}$-while leaving all of the other equations in the system - all of the other functions $g_{j}$ for $i \neq j$-undisturbed.

We are now ready to provide a more precise characterization of an intervention with respect to both deterministic and indeterministic relations. Let $Z_{i}$ be a particular direct cause of $X_{i}$ that is omitted from $\mathbf{V}$ that is, $Z_{i}$ is one of the omitted variables whose combined effect is sum- 
marized by $U_{i}^{\prime}$. Let $U_{i}$ represent the combined effect of all remaining variables in $U_{i}^{\prime}$ except for $Z_{i}$. Then $Z_{i}$ will constitute an (arrow-breaking) intervention with respect to the variable set $\mathbf{V}$ if and only if it meets following conditions:

1. $Z_{i}$ causes $X_{i}$.

2. $Z_{i}$ is not caused by any of the $X \mathrm{~s}$, or other $Z \mathrm{~s}$.

3. $Z_{i}$ does not cause any of the $U \mathrm{~s}$ (nor, as (2) implies, any of the other $Z$ s) and has no causes in common with any of the $U$ s or other $Z$ s.

4. For all $X_{j}$ for $j \neq i$, if $Z_{i}$ or any cause of $Z_{i}$ causes $X_{j}$, then it does so only via a path passing through $X_{i}$.

5. If $X_{i}$ is deterministically caused, then for some range of values of $Z_{i}, \mathbf{z}_{i}^{*}$, if $Z_{i}=z_{i}^{*} \in \mathbf{z}_{i}^{*}$, then $X_{i}=x_{i}^{*}$, regardless of the values of any of the $X \mathrm{~s}, U \mathrm{~s}$, or $Z \mathrm{~s}$. If $X_{i}$ is indeterministically caused, then for some range of values of $Z_{i}, \mathbf{z}_{i}^{*}$, if $Z_{i}=z_{i}^{*} \in \mathbf{z}_{i}^{*} \mathrm{P}\left(X_{i}\right)=\mathrm{P}_{i}^{*}$ regardless of the values of any of the $X \mathrm{~s}, U \mathrm{~s}$, or $Z \mathrm{~s}$. We shall say that $\mathbf{z}_{i}^{*}$ consists of the "on" values of $Z_{i}$. For other values of $Z_{i}, X_{i}$ or $\mathrm{P}\left(X_{i}\right)$ is a function of $p a_{i}$ and other omitted causes, the effect of which is summarized by $U_{i}$.

This characterization is not as complicated as it may look. 2 and 3 require that an intervention be exogenous - it is not caused by any of the other variables in the system and does not have causes in common with any of those other variables. 4 says that if an intervention on $X_{i}$ causes some second variable $X_{i}$, it does so only through its influence on $X_{i}$ and not in some other way. 5 says that an intervention acts as a "switch." When the intervention on $X_{i}$ has an "off" value, the value of $X_{i}$, or in the indeterministic case, $\mathrm{P}\left(X_{i}\right)$ is determined entirely by its parents and the omitted causes whose action is represented by $U_{i}$. When the intervention on $X_{i}$ is "on," the value of $X_{i}$ (or, in the indeterministic case, $\mathrm{P}\left(X_{i}\right)$ ) is determined entirely by the value of the intervention, and the other causes of $X_{i}$ have no influence on it. In other words, an intervention on $X_{i}$ "breaks" all arrows directed into $X_{i}$ except for the arrow coming from the intervention variable.

Let $\mathrm{P}_{\left[Z_{i}=\text { on }\right]}\left(X_{j}\right)$ denote the probability distribution of $X_{j}$ if the value of $X_{i}$ were "set" by an intervention $Z_{i}$ and similarly $\mathrm{P}_{\left[Z_{i}=\text { off }\right]}\left(X_{j}\right)$ denotes $\mathrm{P}\left(X_{j}\right)$ when there is no intervention with respect to $X_{i}$. The generalization of modularity, which we call MOD* can then be expressed as follows:

MOD*. For all $X_{i}$ and $X_{j}$ in $\mathbf{V}(i \neq j)$ if $\mathrm{P}_{\left[Z_{i}=\text { on }\right]}\left(X_{j}\right) \neq \mathrm{P}_{\left[Z_{i}=\text { off }\right]}\left(X_{j}\right)$ then $X_{i}$ causes $X_{j}$.

MOD* formulates a sufficient condition for causation in terms of the results of manipulations. It is also closely linked to CM. Suppose that $X_{i}$ and $X_{j}$ are correlated under an intervention on $X_{i}$. Given the definition 
of an intervention, the correlation cannot be due to (i) some common cause of $X_{i}$ and $X_{j}$ or to (ii) $X_{j}$ causing $X_{i}$. MOD* assumes the only other possibility is (iii) $X_{i}$ causes $X_{j}$. Thus the principle that correlations don't arise spontaneously but always have a causal explanation (at least when interventions are involved) is already implicit in MOD* and in a manipulationist account of causation.

Of course, experiments, especially with a small number of subjects, often show correlations between the values of $X_{i}$ produced by interventions and the values of $X_{j}$ owing to accident or chance rather than because $X_{i}$ causes $X_{j}$. But in such cases, one ordinarily expects that the association will disappear if the experiment is performed repeatedly with a large number of subjects.

What about the possibility that $X_{i}$ and $X_{j}$ remain dependent, although causally unrelated, no matter how one repeats the experiment? One response is to hold that if the relationship between $X_{i}$ and $X_{j}$ looks as though it is causal in the sense that correlations under interventions between $X_{i}$ and $X_{j}$ hold in (what seem to be) representative large populations, then this is all that is required for it to be causal. Alternatively, one may regard doubt at this point about whether $X_{i}$ causes $X_{j}$ as Cartesian or hyperbolic - as uninteresting as skepticism about the existence of the external world. Another response is more methodological: whether or not what is envisioned is logically possible, our experience is that inferences based on MOD* when the dependencies in question hold systematically in representative populations are highly reliable. Furthermore, if one rejects such inferences, one loses the possibility of learning anything about causal relationships from experiments - it is not as though there is some other competing principle one might employ instead. So one should accept MOD*.

3. From the Manipulability Conception to CM. One way of thinking about the connection between the manipulability conception and CM is to think of MOD* as describing what would happen in situations in which there are causal connections and intervention variables are "on" and CM as addressing what would happen in situations in which the same causal connections hold but intervention variables are "off." It is part of ordinary thinking about causation that there will be a systematic connection between these two sorts of situations. When people do experiments, they do them with the expectation that they will learn not just about how the causal agents they manipulate behave in experimental contexts, but how they behave more generally, in other contexts as well.

This connecting assumption can be expressed as follows: conditioning on the parents of a variable simulates the effect of an intervention on that variable in the sense that if $X_{i}$ and $X_{j}$ are dependent conditional on 
$p a_{i}$, then $X_{i}$ and $X_{j}$ will be dependent under an intervention on $X_{i}$. This is the second premise in the argument in Section 4 below. We call it "Indication" since it claims that conditional dependence "indicates" the result of an intervention. In effect, Indication takes the idea that correlations do not arise spontaneously when interventions are "on," an idea which we found to be implicit in MOD*, and extends it to situations in which interventions are "off." CM is an implication of the conjunction of MOD* and Indication.

4. The Argument. Here is the basic outline of our argument for CM:

1. For all $X_{i}$ and $X_{j}$ in $\mathbf{V}(i \neq j)$ such that $X_{i}$ does not cause $X_{j}$, $\mathrm{P}_{\left[Z_{i}=\text { on }\right]}\left(X_{j}\right)=\mathrm{P}_{\left[Z_{i}=\text { off }\right]}\left(X_{j}\right)$ (this is the contrapositive of MOD*).

2. $\forall X_{j}$ in $\mathbf{V}$ distinct from $X_{i}$, if $\mathrm{P}_{\left[Z_{i}=\text { on }\right]}\left(X_{j}\right)=\mathrm{P}_{\left[Z_{i}=\text { off }\right]}\left(X_{j}\right)$, then $X_{j} \perp$ $X_{i} / p a_{i}$ (this is the contrapositive of the condition that we called "Indication" above).

3. If $X_{i}$ does not cause $X_{j}$, then $X_{j} \perp X_{i} / p a_{i}(\mathrm{CM})$ (from 1 and 2).

We assume that 1 is relatively uncontroversial and that those who, like Cartwright, reject CM ought to oppose 2 instead. What then can be said on behalf of 2 ?

Suppose that the full set of parents of $X_{i}$ are held fixed (that is, conditioned on) and assume that $U_{i}^{\prime}$ (the combined effect of the omitted causes for $X_{i}$ ) has no causal connections to any variables in $\mathbf{V}$ apart from those that follow from its being a direct cause of $X_{i}$. Then $U_{i}^{\prime}$ behaves like an intervention (in the sense we have defined) on $X_{i}$. Fixing $p a_{i}, U_{i}^{\prime}$ is an exogenous source of variation in $X_{i}$ that counts as an intervention Thus,

4. If $\mathrm{P}_{\left[Z_{i}=\text { on }\right]}\left(X_{j}\right)=\mathrm{P}_{\left[Z_{i}=\text { off }\right]}\left(X_{j}\right)$, then $X_{j} \perp U_{i}^{\prime} / p a_{i}$.

If one also assumes

$$
\text { 5. If } X_{j} \perp U_{i}^{\prime} / p a_{i} \text {, then } X_{j} \perp X_{i} / p a_{i} \text {. }
$$

one may derive 2. Under determinism or pseudo-indeterminism, 5 is trivial. Since $X_{i}$ is a deterministic function of $p a_{i}$ and $U_{i}^{\prime}$, if $X_{j}$ and $U_{i}^{\prime}$ are independent conditional on $p a_{i}$, it follows that $X_{j}$ and $X_{i}$ are independent conditional on $p a_{i}$. Under indeterminism 5 is more controversial. We think that those who, like Cartwright, reject 2 are more likely to object to 5 rather than 4.

Here is a heuristic argument that (5) should hold under indeterminism: By hypothesis, there are two sources of variation for $X_{i}$ (or $\mathrm{P}\left(X_{i}\right)$ ): $p a_{i}$ and $U_{i}^{\prime}$. If one conditions on $p a_{i}$, one removes it as a source of variation. The only remaining source of variation in $X_{i}\left(\right.$ or $\left.\mathrm{P}\left(X_{i}\right)\right)$ is $U_{i}^{\prime}$. So the only way a correlation could arise between $X_{i}$ and $X_{j}$ (conditional on $p a_{i}$ ) is if 
$U_{i}^{\prime}$ is correlated with $X_{j}$ (conditional on $p a_{i}$ ). Thus if $U_{i}^{\prime} \perp X_{j} / p a_{i}$, it should be the case that $X_{i} \perp X_{j} / p a_{i}$.

This heuristic argument rests on the assumption that a correlation between $X_{i}$ and $X_{j}$ will not arise "spontaneously"- that is, in the absence of some varying cause of $X_{i}$ that is itself correlated with $X_{j}$. In the indeterministic case, where the values of $X_{i}$ and $X_{j}$ are not determined by their causes, this "no spontaneous correlation" assumption rules out the possibility that the variation in the values of these two variables for a fixed value of $p a_{i}$ is coordinated, even though interventions on $X_{i}$ are independent of $X_{j}$.

As an illustration, suppose there are two samples of radioactive material, $S_{1}$ and $S_{2}$. By bombarding $S_{1}$ with neutrons $(C)$, one can increase the probability that the atoms in $S_{1}$ decay, but aside from this, decay in both samples is an entirely random process that depends only on the characteristics of the material in $S_{1}$ and $S_{2}$. Suppose that $(C)$ can take one of two values - on or off - indexed by a random variable $W$ and that one can control $\mathrm{P}(W)$ - that is, one can introduce or remove the neutron source. Let $\mathrm{P}\left(X_{1} / p a_{1}\right)$ and $\mathrm{P}\left(X_{2} / p a_{2}\right)$ be the probabilities for decay of an individual atom in $S_{1}$ and $S_{2}$ respectively, conditional on the characteristics of those atoms, which are summarized by $p a_{1}$ and $p a_{2} .5$ claims that if the random variable $W$ is independent of $X_{2}$ conditional on $p a_{1}$, then $X_{1}$ will be independent of $X_{2}$ conditional on $p a_{1}$. This condition would fail if, as we vary $\mathrm{P}(W), W$ remains independent of $X_{2}$ conditional on $p a_{1}$ (behavior that, given that $W$ behaves like an intervention with respect to $X_{1}$, we would ordinarily take to show that $X_{1}$ does not cause $X_{2}$ ) but it is also true that $X_{1}$ is not independent of $X_{2}$ conditional on $p a_{1}$. If 5 fails in this way, the condition that we called determinism of probabilities will also fail- the joint distribution $\mathrm{P}\left(X_{1}, X_{2}\right)$ will depend not just on the probability of $X_{1}$ conditional on the full set of its causes $\left(\mathrm{P}\left(X_{1} / p a_{1}, W\right)\right.$ ), the probability of $X_{2}$ conditional on the full set of its causes $\mathrm{P}\left(X_{2} / p a_{2}\right)$ and the probability of each set of these causes. Instead there will be an additional dependence in the joint distribution, reflecting the correlation between $X_{1}$ and $X_{2}$, that is not derivable from the probabilities just described (and which indeed seems to come from thin air).

These remarks do not show that violations of (5) are impossible or that they are ruled out by an uncontroversial part of "our concept" of causation. Someone who accepts MOD* yet is convinced that violations of $\mathrm{CM}$ are possible has the option of denying (5) and the Indication condition (2). Nonetheless our discussion identifies just what those who reject $\mathrm{CM}$ are committed to denying and highlights commonplace expectations about causal relationships that are violated when $\mathrm{CM}$ fails to hold.

5. Cartwright's Chemical Factory. Nancy Cartwright (e.g., 2002) asks us 
to imagine a chemical factory that produces two chemicals $A$ and $B$ by an indeterministic process $P . P$ is a common cause of the production of $A$ and $B$ and the only such cause, aside from the possibility of interventions that influence the production of the chemicals directly. In particular, the production of $A$ does not cause the production of $B$ or vice-versa. Let $X$ and $Y$ be indicator variables taking the values 0 or 1 depending on whether $A$ and $B$ are or are not produced. Assume the state of $P$ can also be represented by an indicator variable $C$. As Cartwright describes the case, $\mathrm{P}(X / C)=\mathrm{P}(Y / C)=0.8$ but $\mathrm{P}(X / C . Y)=1$, in violation of $C M$.

Let $Z_{x}$ and $Z_{y}$ be intervention variables with respect to $X$ and $Y$. In the expanded variable set including $Z_{x}$ and $Z_{y}, p a_{x}=\left\{C, Z_{x}\right\}$, and $p a_{y}=\left\{C, Z_{y}\right\}$. Suppose $Z_{x}=$ on. One possibility is that $X$ and $Y$ remain dependent under this intervention. If so, it will be extremely tempting to conclude that $X$ causes $Y$ in accord with MOD* but contrary to Cartwright's description of the example. If $X$ causes $Y$, there is of course no violation of $\mathrm{CM}$.

Although Cartwright (2002) criticizes MOD*, we think she requires it if her example is to be even a prima facie counterexample to CM. Only by adopting MOD* and a corresponding interventionist interpretation of causation can one tell a consistent story about her example. Accordingly, in what follows, we will assume in accord with MOD* that when $Z_{x}=$ on, $X$ and $Y$ are independent conditional on $C$. However, when $Z_{x}=$ off, $X$ and $Y$ are dependent conditional on $C$.

Under this construal, MOD* holds but Indication (premise 2) fails, as of course does CM. Although the distribution of $Y$ is the same when the intervention variable for $X$ is on as when it is off (since, by hypothesis, $X$ and $Y$ are no longer correlated when the intervention is "on"), it is not true that $X \perp Y / C$. Moreover, of the two premises - 4 and 5-that jointly imply Indication, it is, as suggested above, 5 that fails rather than 4. Construing $U_{x}^{\prime}$ as an intervention variable, $U_{x}^{\prime}$ is independent of $Y$, conditional on $C$ for both the on and off values of $U_{x}^{\prime}$, as 4 requires, but 5 is violated.

What is odd about the example is not that certain variables are independent or conditionally independent for some values and not for others. There is nothing perplexing about this. Instead what is odd is that conditional independence relations hold or fail to hold for the same values of variables depending on how those values are produced. When the values of $X$ and $Y$ are caused by $C$, and the intervention variables $Z_{x}$ and $Z_{y}$ are "off," $X$ and $Y$ are conditionally dependent. When $X$ and $Y$ have those very same values but those values are instead caused by the "on" values of $Z_{x}$ or $Z_{y}, X$ and $Y$ are conditionally independent. There is no systematic connection between the conditional independence relations that hold when interventions are on and the relations that hold when inter- 
ventions are off. This violates standard expectations, described in Section 3 , about the connection between the behavior of causes in experimental and nonexperimental situations.

6. Conclusion. In her discussions of $\mathrm{CM}$, Cartwright claims not just that violations of $\mathrm{CM}$ are logically possible but that such violations "are to all appearances widespread" even in the macroscopic, non-quantummechanical realm. (Her discussion makes it clear that the violations she imagines are over and above any that may be due to the defeating conditions described in Section 1 above.) This is a surprising claim. The chemical factory is imaginary and Cartwright has not produced any reallife macroscopic examples in which CM fails. This suggests (we do not claim that it conclusively establishes) that violations of $\mathrm{CM}$ violate widely shared expectations about the relationship between causes, interventions, and probabilities. In this essay we have suggested what some of these expectations are. People do not expect spontaneous correlations, and they do expect that there will be systematic relationships between (in)dependence relationships when intervention variables are off and when they are on, so that they can use each kind of information to learn about the other. ${ }^{1}$ As in the case of MOD*, these expectations may not be logically inviolable but they seem to be highly reliable in actual application, and they seem to be required if one is able to learn about causal relationships from nonexperimental evidence in the absence of detailed background information.

\section{REFERENCES}

Cartwright, Nancy (2002), "Against Modularity, the Causal Markov Condition and Any Link between the Two: Comments on Hausman and Woodward", British Journal for the Philosophy of Science 53: 411-453.

Gopnik, Alison, Clark Glymour, David Sobel, Laura E. Schulz, Tamar Kushnir, and David Danks (2004), "A Theory of Causal Learning in Children: Causal Maps and Bayes Nets", Psychological Review 111(1): 3-32.

Hausman, Daniel, and James Woodward (1999), "Independence, Invariance, and the Causal Markov Condition”, British Journal for the Philosophy of Science 50: 521-583.

Hitchcock, Christopher (2001), "A Tale of Two Effects", The Philosophical Review 110: 361396.

Spirtes, Peter, Clark Glymour, and Richard Scheines (2000), Causation, Prediction, and Search, 2nd ed. New York: MIT Press.

Woodward, James (2003), Making Things Happen: A Theory of Causal Explanation. Oxford: Oxford University Press.

1. These claims are testable in principle: put people in situations in which such expectations are violated and see how they react. If Cartwright is correct, they will not find such violations surprising or puzzling, since they are common in real life. For some preliminary evidence (involving young children) to the contrary, see Gopnik, Glymour, Sobel, Schulz, Kushnir, and Danks 2004. 